\title{
Research on Risk Decision of Overseas Energy Investment Project Based on Grey Clustering Analysis
}

\author{
Zhenhua Zhang \\ Business School, Jiujiang University, Jiujiang, China \\ zh_ang_zhenhua@126.com
} Keywords:Ioan transaction; accuracy; grey clustering analysis; information control; risk indicator
system

\begin{abstract}
With the development of economic globalization, the loan transaction of overseas projects that accepted by domestic banks is increasing. In the treatment of overseas project's lending decisions, the traditional risk decision evaluation can't meet the need of accuracy and promptness of the decision-making, so the usage of more scientific and reasonable analytical method is imperative. Therefore this paper was through the method of grey clustering analysis to control the information of construction plan, and it got the risk indicator system to construct the decision-making model. And this paper put the accurate results as data set of the historical cases, it provided real-time basis to the next analysis and decision, in this way it can assist the efficient implement of overseas energy investment project.
\end{abstract}

\section{Introduction}

In early August of last year, China's development and reform commission announced the newly approved five overseas energy investment projects, and the project types included petroleum, natural gas, liquefied coal-bed methane. The locations of whole project contained Asia, Africa, Oceania and other areas. The ratification of multiple projects means that the investments of overseas resources projects in our country enter into the intensive period, and China further speeds up the steps of seeking energy resources in the global scope[1]. How to have the accurate and efficient evaluation of overseas projects' credit risk and make the corresponding loan program is a very urgent task of the domestic bank.

Every project's investment risk of the energy investment is different, and safety situation of the coal industry's development is very serious, so the risks of coal, oil and gas are relatively high, while the risk of power investment is lower than the risk of industrial investment[2]. Development of diversified energy promotes the development of new energy, and its investment risk is relatively lower than other resources.

\section{Grey clustering analysis}

Grey clustering analysis is one of grey system theory's contents, it is the method that based on the whitened function of grey number, the values of the clustering objects' different clustering indexes have induction and arrangement[3].According to the grey class, it can determine that the clustering belongs to grey system statistical method of which kind of grey classes. Although the method is based on the mathematical model, it is also different from general quantitative methods[4]. Clustering analysis on the whitened function of grey class is mainly used to check whether the result belongs to different categories and settings, and then it provides the differential treatment. The right of grey clustering analysis is mainly used for similar factors, and it can make the complex system have integration and simplification. The merit of grey clustering analysis is that there does not have special requirements for the samples' distribution of clustering objects, and it only needs a simple calculation process to get more useful information. The whitened function can be expressed as[5]:

$$
f_{2}\left(d_{i j}\right)=\left\{\begin{array}{c}
\frac{x-a}{b-a}, x \in[a, b] \\
\frac{c-x}{c-b}, x \in[b, c] \\
0, x \notin[a, c]
\end{array}\right.
$$


$\lambda_{j k}$ is the threshold value of $f_{j k}, \lambda_{j k}$ is the objective threshold value and $f_{j k}$ is the relative threshold value.

\section{Information control system of construction plans}

A. Risk analysis on loan of overseas energy investment project

As transnational investment, the risk of overseas energy investment projects is higher than the average level of other domestic investment projects. Here the overseas investment risk can be divided into four primary risks: country risk, business risk, legal risk and bank credit risk, and it also has 19 secondary risks, the specific is shown in Table 1.

TABLE I. THE RISK TYPES OF OVERSEAS PROJECTS

\begin{tabular}{|l|l|l|}
\hline \multirow{2}{*}{ Primary risk } & Secondary risk & Reasons of risk \\
\hline \multirow{5}{*}{ Country risk } & Sovereign risk & \multirow{2}{*}{ Political factors, legal norms factors, conflicts of economic policy between the countries } \\
\cline { 2 - 3 } & Transfer risk & Government constraints \\
\hline \multirow{5}{*}{ Business risk } & system risk & Instability \\
\cline { 2 - 3 } & Investment risk & Error of assessment results, professional ethics, a large number of liquid resources \\
\cline { 2 - 3 } & Assessment risk & The stock quota control, investment risk \\
\cline { 2 - 3 } & Financing risk & Boycott of risk \\
\cline { 2 - 3 } & Antitrust risk & The overall planning omission, market change \\
\cline { 2 - 3 } & Operating risk & The synergistic effect, system integration, inappropriate scale and range \\
\cline { 2 - 3 } & Integrated Risk & Antitrust rules, international regulations, investment terms, compliance risk \\
\hline \multirow{5}{*}{ Bank credit risk } & Special legal risks & Changes in the lending rate \\
\hline & Market risk & Capacity factors and ethical considerations of bank's internal staff \\
\cline { 2 - 3 } & Compliance risk & Changes in currency exchange rates \\
\cline { 2 - 3 } & Exchange rate risk & Changes in interest rates \\
\cline { 2 - 3 } & Interest rate risk & \\
\hline
\end{tabular}

B. Analysis on implementation plan of overseas energy investment project

In the process of the traditional implementation of overseas project's loan, the banks have five experience loans, and they are plan A, plan B, plan C, plan D and plan E[6].

Plan A: It should build overseas subsidiaries, and subsidiaries are responsible for the specific operation of investment project. The parent company is as a credit to become the AAA level customer of the bank, and these loans are limited in the range of bank credit.

Plan B: It should build overseas subsidiaries, and subsidiaries are responsible for the specific operation of investment project. The subsidiary is as a borrower, and the subsidiary has pledge of assets and the guarantee structure.

Plan C: On the basis of plan B, the parent company will build wholly owned subsidiary in the foreign countries, subsidiary is responsible for collection and operation. The subsidiary is as borrower, assets of subsidiary are mortgaged, and all rights of the parent and subsidiary company are mortgaged and pledged.

D plan: The products of the company are as mortgage of subsidiary, they are not the equity of subsidiaries. The revenue of auxiliary product is used to repay bank debt. It not only makes the cash flow convenient, but also avoids the legal conflict between different countries.

E plan: Subsidiary has practical power, and such as the local jurisdiction is used as bank mortgage, the income of subsidiary is used to pay for the bank loans.

According to the five kinds of loan schemes, plan A and plan B are time-consuming and they can not guarantee the safety of fund, so plan $\mathrm{C}$ is put forward on the basis of plan A and plan B. Plan C has low risk, but it will improve the parent company's responsibility to a very important position, and this reduces the bank's income. Plan D and plan E are actually the supplements of plan B, at same time the purpose is to meet some overseas energy investment projects, such as coal, electricity and mining. The implementation schemes of the five kinds of loans are shown in Table 2, and the table also has the survey of the borrower and risk. 
TABLE II. COMPARISON BETWEEN THE FIVE KINDS OF LOAN SCHEMES

\begin{tabular}{|c|l|l|l|}
\hline Capital risk of loan programs & \multicolumn{1}{|c|}{ Capital operation } & \multicolumn{1}{|c|}{ Borrower } & \multicolumn{1}{c|}{ Guarantors } \\
\hline Plan $\boldsymbol{A}$ & Subsidiary & Parent company & Parent company \\
\hline Plan $\boldsymbol{B}$ & Subsidiary & Subsidiary & Subsidiary \\
\hline Plan $\boldsymbol{C}$ & Subsidiary & Subsidiary & Parent company and subsidiary company \\
\hline Plan $\boldsymbol{D}$ & Subsidiary & Subsidiary & Subsidiary \\
\hline Plan $\boldsymbol{E}$ & Subsidiary & Subsidiary & Subsidiary \\
\hline
\end{tabular}

According to the complicated situation of overseas investment project loans, the most appropriate loan programs should make the accurate and reasonable evaluation of loan project.

\section{Establishment of project decision-making models based on the grey clustering analysis}

Establishment of project decision-making models that based on the grey clustering analysis mainly is divided into two steps, and they are "establishment of the risk index system" and "grey clustering analysis of historical case"[7]. The standard way of establishing risk indicator system is changed the original classification of assets into the grey clustering analysis method. "Grey clustering analysis of historical case" is at the situation of inputting the historical cases, and according to the grey clustering analysis it has construction of the risk indicator system. It is based on the historical case analysis to determine the corresponding loan programs, and then it can carry out the implementation plan of the decision-making of project loan.

C. Establishment of risk indicator system

1) Principle of establishing the risk indicator system

Evaluation indicator's structure model of loan credit is directly related to the results of the evaluation. Evaluation index system is independent, objective, systematic and effective. During the operation, if the selection indexes are too much, it will appear redundancy and cause repeated interference index; if the selection indexes are too few, they may not represent all the indexes, and they will lead to inadequate situation. It introduces the structure model of loan credit to have comprehensive evaluation, in this way it can establish a scientific and reasonable evaluation index system, at the same time the system can reflect the loan credit of the whole overseas project of merger and acquisition.

2) Structure of risk index system

The original risk factors can be seen in Table 1, when it is based on grey clustering analysis method to establish the risk indicator system, the three kinds of grey number "high", "medium” and "low" are used to respectively express harmful degree of different risk indicators, the Delphi method is put as a quantitative method. Detailed risk indicator system's construction process of grey clustering analysis is shown as follows:

(1) It structures matrix of whitened function, then the indexes have clustering. Clustering index function of each object can establish matrix of whitened function. ${ }^{d_{i j}}$ is whitened number of the first i clustering object that related to the first $\mathrm{j}$ clustering index, and D is the sample matrix[8].

$$
D=\left[d_{i j}\right]=\left[\begin{array}{cccc}
d_{11} & d_{12} & \cdots & d_{1 m} \\
d_{21} & d_{22} & \cdots & d_{2 m} \\
\cdots & \cdots & \cdots & \cdots \\
d_{n 1} & d_{n 2} & \cdots & d_{n m}
\end{array}\right]
$$

(2) It is through the whitened function to determine the grey clustering coefficient.

$\eta_{j k}$ is the clustering right of grey class $\mathrm{k}$ of the same index object i. $\eta_{j \mathrm{k}}$ is the result that based on two aspects' calculation.

With the same dimension and standard, there has:

$$
\eta_{j k}=\frac{\lambda_{j k}}{\sum_{j=1}^{m} \lambda_{j k}}
$$


If the clustering index is different from the dimension, the number of different indicators in the samples is very big, and $\eta_{j k}$ can't be calculated, otherwise it is easy to have deviation.

When the absolute values of clustering coefficient's unit and index are very different, there has[9]:

$$
\gamma_{j k}=\frac{S_{j k}}{S_{j}}
$$

In the above formula, ${ }^{S k}$ is grey number (standard) of grey class $\mathrm{k}$ of the index $\mathrm{j}$. The grey clustering right $\eta_{j k}$ can be calculated through the following formula[10]:

$$
\eta_{j k}=\frac{\gamma_{j k}}{\sum_{j=1}^{m} \gamma_{j k}}
$$

The clustering right $\eta_{j k}$ uses the Delphi method to give different rights to the indexes, and this makes the weighted and adjusted clustering right more scientific and reasonable.

(3) Calculation of grey clustering coefficient

It has to do a whitened process to measure the samples, and finds out the threshold value of grey type. Then grey index of the first $\mathrm{j}$ index clustering is $\sum_{i=1}^{n} \lambda_{i j}$. Grey clustering coefficient $\sigma_{i j}=\sum_{i=1}^{n} f_{i j}\left(d_{i j}\right) w_{i j}, \sigma_{i h}$ reflects the first i clustering object, it belongs to the grey class $\mathrm{j}$, and it gets the clustering row vector $\sigma_{i}=\left(\sigma_{i 1}, \sigma_{i 2} \cdots \sigma_{i s}\right)$. S is as a grey model. $\sigma_{c}$ is grey clustering decision matrix, the formula is as follows[11]:

$$
\sigma_{c}=\left[\begin{array}{c}
\sigma_{1} \\
\cdots \\
\sigma_{n}
\end{array}\right]=\left[\begin{array}{ccc}
\sigma_{11} & \cdots & \sigma_{1 k} \\
\cdots & \cdots & \\
\sigma_{n 1} & \cdots & \sigma_{n k}
\end{array}\right]
$$

If $\sigma_{i k^{*}}=\max _{1 \leq k \leq K}\left\{\sigma_{i k}\right\}=\max \left\{\sigma_{i 1}, \sigma_{i 2}, \cdots, \sigma_{i k}\right\}$, then we can know that the object $\mathrm{i}$ belongs to the grey class $\mathrm{k}$

That is to say, in the clustering row vector it finds the biggest clustering coefficient. So it can complete the establishment of project risk indicators of the grey clustering analysis method. The risk indicator system of grey clustering analysis can be seen in Figure 1.

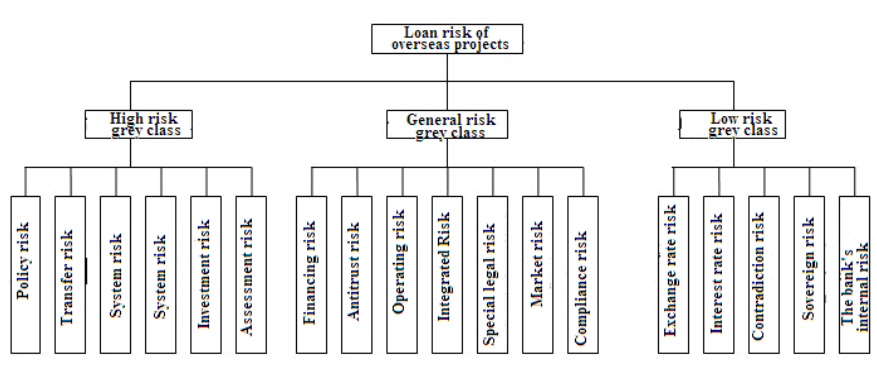

Figure1.Risk indicator system of grey clustering analysis

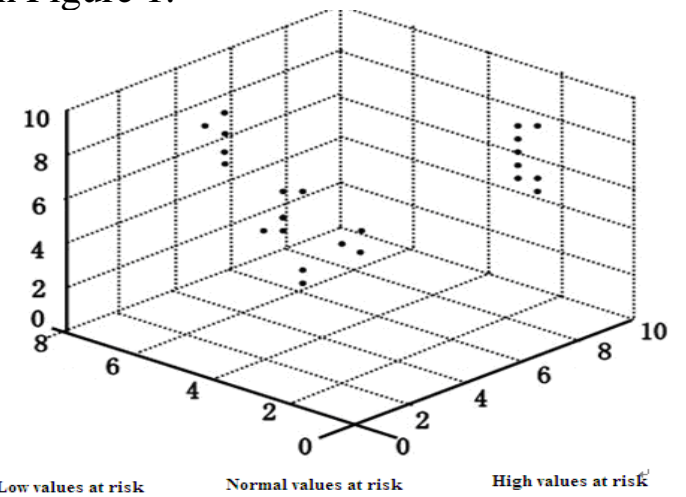

Figure2.Result of clustering analysis

D. Analyses on history loan cases based on the grey clustering method

This step can calculate the values at risk of three kind of grey classes, each clustering case has clustering in three dimensional space, and then it is based on the three dimensional clustering to get implementation plans of five kinds of loans.

After the analysis of grey clustering, the values at risk of "high risk", "general risk" and "low risk" these three kinds of grey classes have clustering, and the results can show difference and contact of the five kinds of loan programs[12]. This paper selected 30 cases of overseas project loan in accordance with the clustering analysis method, and they include the coal, electric power, industry, infrastructure, culture education, public health and other aspects. The actual status of the 
corresponding loan programs can be seen in Table 3, and the results of clustering analysis are shown in Figure 3.

Figure 3 shows clustering analyses of the 30 cases, “A”, "B”, "C", "D" and "E” are respectively expressed the clustering centers of the five kinds of overseas project loan schemes, their special coordinates are A $(5,5,5), \mathrm{B}(9,2,7), \mathrm{C}(1,6,9), \mathrm{D}(8,6,1)$ and $\mathrm{E}(2,4,3)$. The result of clustering analysis in Figure 2 is the decision model of overseas project loan.

The risk decision of overseas energy investment project that based on grey clustering analysis method is the establishment of overseas project decision, then risk indicators of all kinds of decisionmaking are calculated, and the "high", "medium", and "low" three grey classes of each project are compared with each other. In Figure 2 , we can look for the most suitable clustering center in the five kinds of clustering centers, and then it can be considered to be the most suitable implementation plan that has minimal risk.

TABLEIII. SELECTION OF HISTORY CASES

\begin{tabular}{|l|l|l|l|l|l|}
\hline & Case A & Case B & Case C & Case D & Case E \\
\hline The number of cases & 10 & 10 & 5 & 3 & 2 \\
\hline
\end{tabular}

\section{Conclusion}

Along with the development of national economy and the strengthening of comprehensive national strength, in China the requirement of seeking the energy resources in the global environment is increasingly urgent. The banks and government pay attention to the problems that how to have accurate and efficient evaluation of overseas project loan risk and make the corresponding loan programs. According to the past experience, decision-making work of traditional overseas project is mainly calculated the experimented by related persons of bank loans. But compared with traditional methods, risk decision of overseas energy investment project that based on grey clustering analysis method can overcome the shortcomings of traditional methods, at the same time it also can improve the accuracy and reliability of risk decision.

\section{References}

[1] Liang Xu. Application of grey clustering analysis method in the study of the detector optimization [J].Journal of Changchun College of engineering, 2009(07):36-39。

[2] LiPing Yang. Study on credibility of grey clustering analysis in decision-making [J]. Science press, 2012:321-324

[3] Sifeng Liu, Zhigeng Fang. Theory and application of grey system [M].Science press, 2010(11):87-80.

[4] YanLing Li. Comprehensive evaluation model of ecological environmental quality based on the grey clustering analysis method [J].Surveying and mapping science, 2011(05):51-54

[5] Jianzhong $\mathrm{Xu}, \mathrm{Li} \mathrm{Li}$. Using the grey clustering method to determine the third industry internal development strategy [J]. Statistics and decision, 2010 (14):95-97

[6] Julong Deng. Basic method of grey theory [M].Huazhong university of science and technology press, 2010(06):22-25

[7] Desheng Yi. Grey theory and method [M]. People's education press, 2011:279-281

[8] Peng Wang. Detailed explanation of new five overseas energy investment projects [J].Chemical management, 2011(10):35-38

[9] Sheng Chang. Application of grey clustering method in the Shengzhong reservoir's eutrophication evaluation [J].Journal of agricultural environment science, 2008(6):96-99

[10] Yun Wang. rey clustering decision of science and technology journals [J]. Journal of intelligence, 2011 (09):68-70

[11] Linglong Kong, Hongkuan Liu, Guohui Li. The risks and countermeasures of foreign investment [J].National development and reform commission, 2010(03):35-36;

[12] Tingbo Wang, Shichao Xu. Network security situation assessment method based on the analytic hierarchy process [J]. The Computer knowledge and technology, 2008(10): 79-81 